\title{
Kids am Kap
}

\section{Vor 20 Jahren schüttelten die}

Südafrikaner das Joch der Apartheid ab.

Wo steht das Land heute? Unsere Autorin

hat ein PJ-Tertial in einer Kinderklinik in

Kapstadt absolviert und schildert ihre

Eindrücke aus medizinischer Sicht.

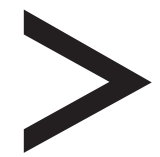

Eine Durchsage über Funk. Wie angekündigt, landet kurz darauf ein Helikopter auf dem Landeplatz der Kinderklinik. Die Rettungssanis und eine Notärztin in roten Overalls bringen einen vierjährigen Jungen auf einer Trage in den ER. Die aufgelöste Mutter erzählt, ihr Sohn habe am Kabel des Wasserkochers gezogen und sich dabei an Arm, Hals und Körper verbrüht. Das Rettungsteam hat bereits einige i.v.-Zugänge gelegt und die Sedierung und Schmerzmedikation begonnen. Nun übernehmen Pfleger die Versorgung: Sie entfernen die Kleidung und schätzen Ausmaß und Tiefe der Verbrennungen. Die Wunden decken sie mit „Burnshields“ ab - einzeln verpackte Kompressen, die mit Wasser und Teebaumöl getränkt sind. Mittlerweile ist der diensthabende Arzt aus der Burns-Unit dazugestoßen. Während die Geräte zur Überwachung der Vitalfunktionen im Hintergrund piepsen, übergibt die Notärztin

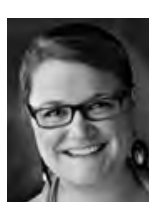

\section{Autorin}

Laura Olbrich ging nach ihrem

Chirurgie-Tertial in Südafrika auch noch nach Ghana, wo sie ihr

Pädiatrie-Tertial absolvierte. Seit Herbst letzten Jahres hat sie ihre Approbation und arbeitet jetzt in der Kinderklinik der Uniklinik Düsseldorf. Kontakt: laura.olbrich@googlemail.com 
den Fall an ihren Kollegen. Eine Krankenschwester kümmert sich um die Mutter. Sobald der Junge stabilisiert und der Unfallhergang klar ist, wird der Flüssigkeitsverlust und -bedarf mit der Parkland-Formel* berechnet und die Leitung der Burns-Unit über den neuen Patienten informiert.

\section{> Als PJler im Red Cross}

Angesichts dieser sauber getakteten Rettungskette mag sich mancher verwundert die Augen reiben. Befinden wir uns hier nicht doch in einer europäischen oder amerikanischen Klinik? Antwort: Nein, wir sind tatsächlich in Afrika. Genauer: im Red Cross War Memorial Children's Hospital in Kapstadt, dem einzigen reinen Kinderkrankenhaus der Maximalversorgung südlich der Sahara. Hier - vor allem in der Kinderorthopädie und der Trauma-Abteilung - habe ich mein Chirurgie-Tertial verbracht. Die Qualität der Medizin im Red Cross ist vergleichbar mit einer deutschen Klinik. Auch die Palette der Krankheitsbilder ist ähnlich. Und doch gibt es eklatante Unterschiede: Besonders in der Trauma-Abteilung habe ich vieles sehen können (oder müssen), was es bei uns in diesem Ausmaß nicht gibt. So sind Verbrennungen hier an der Tagesordnung und stehen an dritter Stelle der kindlichen Todesfälle. Besonders im Winter verbrühen sich die Kinder häufig, denn in den riesigen Slums wird oft über offenem Feuer gekocht. Zudem verbreiten sich Brände durch die enge Bauweise rasant. Hinzu kommen die wirklich extrem häufigen und schweren Verkehrsunfälle und Übergriffe auf Kinder: Alleine in unserer Abteilung werden um die 100 neue Fälle sexuellen Missbrauchs pro Jahr registriert. Besonders hier, wo die HIV-Epidemie so heftig grassiert, ist dies nur schwer zu verdauen.

Ganz anders war das Bild in der Kinderorthopädie: Als eine spezielle Fachrichtung wurden uns Patienten aus ganz Südafrika überwiesen. Neben typischen Krankheitsbildern, wie etwa Hüftgelenksdysplasien, M. Perthes und ungewöhnlichen „Kolibris“ wie
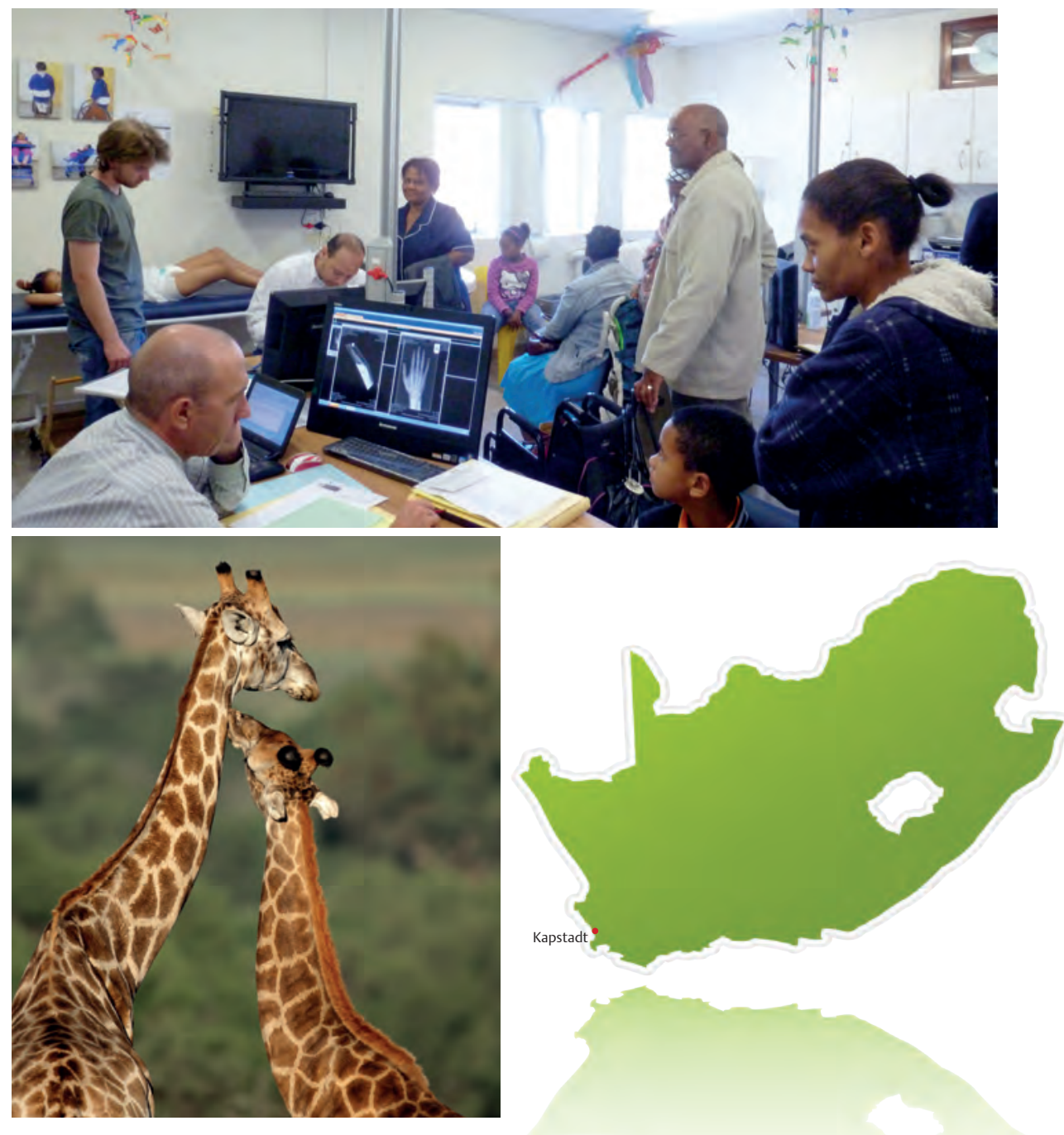

> Oben: Ein Blick in die orthopädische Sprechstunde des „Red Cross“. Typisches Bild hier: Hellhäutige Ärzte behandeln vornehmlich dunkelhäutige Patienten. Der Grund: Zu wenig Schwarze studieren Medizin.

> Unten: Südafrika mag viele Probleme haben, sein Reichtum an natürlicher Schönheit jedoch ist atemberaubend. Allein von diesem langhalsigen Tier leben in den Nationalparks des Landes ungefähr 12.000 Exemplare.

der Aplasie von langen Röhrenknochen bei Neurofibromatose, hatten wir regelmäßig Patienten mit Epiphysiolysis capitis femoris und Morbus Blount, der in der schwarzen Bevölkerung gar nicht so selten ist. Auch Tuberkulose-Patienten waren häufig. Da bei TBC aufgrund der langen Behandlung mit Tuberkulostatika eine hohe Compliance erforderlich ist, lagen diese Kinder oft Monate bei uns.

\section{> Post-Visite mit Chef und Kaffee}

Wie in den meisten größeren Krankenhäusern Südafrikas finden sich auch im Red Cross viele internationale Studenten - auch deutsche PJer. Trotzdem war die Stimmung gegenüber den jungen Medizinern stets wertschätzend und entspannt. Abhängig vom jeweiligen Arzt konnte ich sowohl im ER mithelfen (z. B. beim Gipsen und Nähen) als auch im OP in der Orthopädie - meistens als zweite Assistenz, manchmal aber auch als erste. Besonders bei den Orthopäden herrscht ein sehr lockerer und netter Umgang. Nach der Visite lud uns unser Chef Dr. Dixpeek täglich zum Kaffeetrinken in der Cafeteria ein. Einer der Assistenzärzte war gerade dabei, sein eigenes Bier zu entwickeln - und war sehr an 


\section{$>$ Theoretisch hat in Südafrika jeder Zugang zu allen Gesundheits- leistungen. Faktisch sind viele jedoch ausgeschlossen. <}

meiner "fachfrauischen“ Meinung als Deutsche interessiert. Es wurde aber auch viel Wert darauf gelegt, dass die Studenten was lernen. Wer Interesse zeigte, dem wurde viel erklärt. Aufgrund der Bestimmungen unseres Landesprüfungsamtes war ich als offizielle Studentin der Uni Kapstadt eingeschrieben. So konnte ich an Lehrveranstaltungen teilnehmen - z. B. an der zweiwöchigen Studentenrotation durch die chirurgischen Abteilungen. Dafür musste ich natürlich Studiengebühren zahlen: Anstatt im Monat 400 Euro für das PJ zu bekommen, musste ich den gleichen Betrag investieren.

\section{$>$ Das Krankenhaus als Spiegel der Gesellschaft}

Angesichts der Professionalität, mit der die Abläufe im Red Cross gemanagt werden, vergisst man leicht, in welchen Schwierigkeiten das Land steckt. Kapstadt ist ein Ort, an dem die Gegensätze, die Südafrika prägen, besonders heftig aufeinanderprallen: Auf der einen Seite gehört die Stadt zu den Modemetropolen mit riesigen Shoppingzentren, Edelboutiquen und Fotoshootings mit Topmodels, auf der anderen Seite gibt es riesige Elendsviertel. Die Spannungen zwischen den ethnischen Gruppen treten hier immer wieder zutage. Wie wenig die Südafrikaner über 20 Jahre nach Ende der Apartheid zu einer Einheit verschmolzen sind, merkt man schon daran, dass man nach wie vor oft an der Hautfarbe ablesen kann, aus welchem Viertel jemand kommt oder zu welcher Einkommensschicht er gehört.
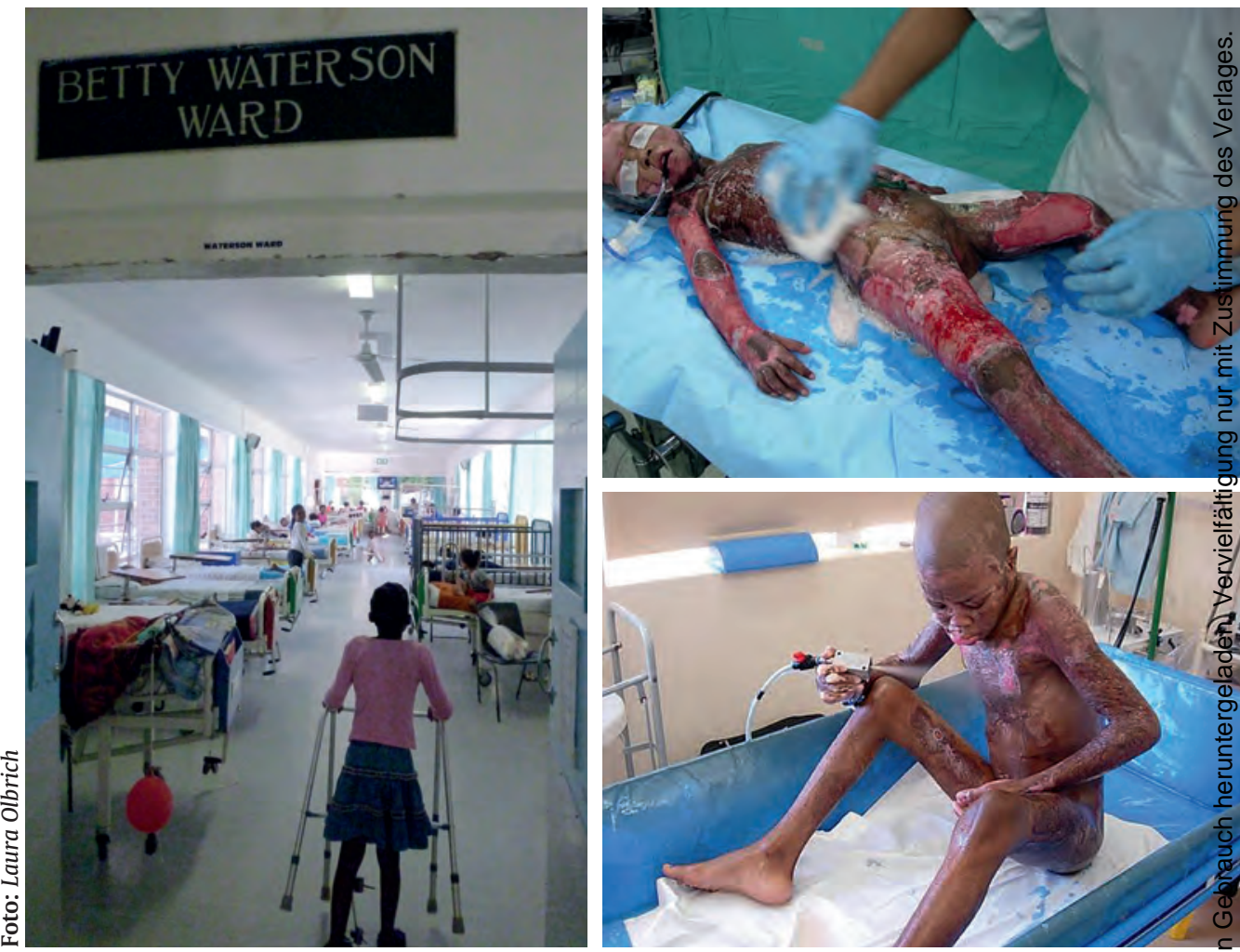

> Links: Die „girls ward“ im Maitland Cottage Home. Hier liegen die orthopädischen Patienten des Red Cross. Rechts: Bei schweren bzw. großflächigen Verbrennungen hat die Prävention von Infektionen höchste Priorität. Deswegen werden die Kinder unter Sedierung regelmäßig gewaschen - oder sie waschen sich selbst.
Auch unsere Klinik ist diesbezüglich keine heile Welt. Wenn man Ärzte und Patienten vergleicht, sieht man die Spaltung der Gesellschaft recht deutlich: Die Mediziner sind weiß oder kommen aus Indien. Die Patienten sind farbig oder schwarz. Obwohl sie die Mehrheit der südafrikanischen Bevölkerung stellen, sind

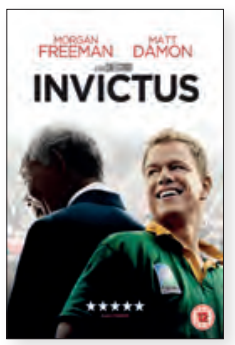

\section{Mitmachen und gewinnen!}

Lange wurden die „Springbokke“, Südafrikas Rugby-Nationalteam, wegen der Apartheidpolitik des Landes boykottiert. 1995 - nun als Team einer freien Nation - wurden sie sofort Weltmeister im eigenen Land. Den Pokal überreichte Mandela persönlich. Wir verlosen drei DVDs mit dieser von Clint Eastwood unter dem Titel „Invictus“ verfilmten Geschichte. Teilnahme unter: www.thieme.de/ viamedici/zeitschrift/spezial. Stichwort „Invictus“. Teilnahmeschluss: 5.5.2014 mir verhältnismäßig wenige dunkelhäutige Ärzte begegnet. Bei den Studenten ist die Verteilung ausgeglichener - auch weil es mittlerweile Quoten und Programme gibt, um eine gerechtere Vergabe zu gewährleisten.

Der Staat gibt sich durchaus Mühe, das soziale Gefälle auszugleichen. Da die Zuzahlungen zu den staatlichen Gesundheitsdiensten abhängig vom Einkommen gestaffelt sind, hat in Südafrika theoretisch jeder Zugang zur Gesundheitsversorgung. Arbeitslose und Geringverdiener zahlen überhaupt nichts. Es gibt zwar einen großen privaten Sektor, der vor allem die Bedürfnisse der Besserverdiener abdeckt. Für die ganz speziellen Fragestellungen gehen aber auch diese in universitäre Einrichtungen, die allen offen stehen. Die medizinische Versorgung in den großen Zentren ist hervorragend. Be- 
sonders positiv ist die Situation im Red Cross, denn der „Children's Hospital Trust“ (eine klinikeigene Stiftung) und viele Einzelpersonen bemühen sich sehr erfolgreich um Spenden und Unterstützung - die Spendertafeln überall zeugen hiervon. So hat es während meines Aufenthaltes nie an etwas gefehlt, sei es apparative Diagnostik, Medikamente oder Personal. Zudem zählt das Haus zu den renommiertesten afrikanischen Referenzzentren in ziemlich allen pädiatrischen Belangen, und es befinden sich regelmäßig Patienten aus dem Ausland hier in Behandlung, teils selbst finanziert, teils durch Spenden ermöglicht.

\section{> Der Mangel abseits der Zentren}

Faktisch sind viele Menschen von dieser hohen medizinischen Qualität in den gut ausgestatteten Zentren aber leider nahezu ausgeschlossen. Sie leben einfach zu weit weg, und die Transportkosten sind so hoch, dass sie gar nicht erst zu diesen Kliniken kommen. In den ländlichen Gebieten sind die ersten Anlaufstellen bei einer Erkrankung die Gesundheitsposten. Diese sind oft nur mit Krankenpflegern besetzt, da, ähnlich wie bei uns auch, in Südafrika immer weniger Mediziner auf dem platten, leeren Land wohnen wollen. Vom Gesundheitsposten werden die Patienten gegebenenfalls an die nächsthöhere Versorgungsstufe in einer kleineren Klinik überwiesen, wo sie dann erstmals von einem Arzt gesehen werden. Dann erst geht es weiter in die nächste größere Stadt oder in ein größeres Zentrum, sodass eine „Patientenkarriere“ schon mal sehr lange sein kann. In den Townships sind die Gesundheitsposten und Kliniken oft völlig überlaufen. Es fehlt an Personal, Platz und Materialien. Oft müssen selbst Notfälle Stunden warten - obgleich Ärzte und Pflegekräfte ihr Bestes geben. Weitere Probleme ergeben sich daraus, dass viele Patienten erst Hilfe aufsuchen, wenn die Krankheit schon weit fortgeschritten ist, sei es aufgrund von finanziellen Engpässen, fehlendem Gesundheitsbewusstsein oder einfach Angst vor einer „brandmarkenden“ Dia- gnose wie HIV. Diese Krankheit ist nach wie vor ein Stigma. Das gilt auch für die Tuberkulose, weil sie häufig in Verbindung mit HIV auftritt.

\section{> Fazit: Positiv mit Frustanteilen}

Diese Probleme sind nicht nur im Gesundheitssektor, sondern so ziemlich in jedem Bereich des Lebens zu fühlen. Die Sicherheitslage ist prekär. Ich hatte permanent das Gefühl, auf der Hut sein zu müssen. Auch das Verhältnis zwischen den Rassen habe ich häufig als bedrückend empfunden. Selbst zwischen Freunden knallt es immer mal wieder - auch bei nur kleinsten Missverständnissen zum Thema Hautfarbe. Meine nigerianische Mitbewohnerin hat mir z.B. erzählt, dass sie merkwürdig angesehen wurde, als sie in einem Café ihren Kaffee in

\section{Selbst zwischen Freunden knallt es immer wieder mal} wegen des Themas Hautfarbe.

ihrer westafrikanischen Unbefangenheit mit den Worten „I like it black“ bestellte. Und auch ich habe jedes Mal komische Blicke geerntet, wenn ich in meinem nicht ganz korrekten Denglisch davon schwärmte, wie bunt („coloured“) ich das Krankenhaus finde ...

Wenige Monate nach meiner Rückkehr nach Deutschland starb Nelson Mandela. Es gibt in Südafrika Pessimisten, die fürchten, ihr Land werde nun - da der große Brückenbauer fehlt - noch weiter auseinanderdriften. Schaut man sich an, wie gespalten die Nation jetzt schon ist, könnte man in der Tat nachdenklich werden. Ich sehe es aber eher wie Mandela selbst, der in seiner Autobiografie schrieb: „Ich bin Optimist. (...) Zum Optimistischsein gehört, das Gesicht der Sonne zuzuwenden und immer vorwärtszugehen. Es gab viele dunkle Stunden, in denen mein Glaube an das Gute auf die Probe gestellt wurde. Aber ich wollte und konnte mich nicht aufgeben. Denn wer diesen Weg geht, hat schon verloren.“

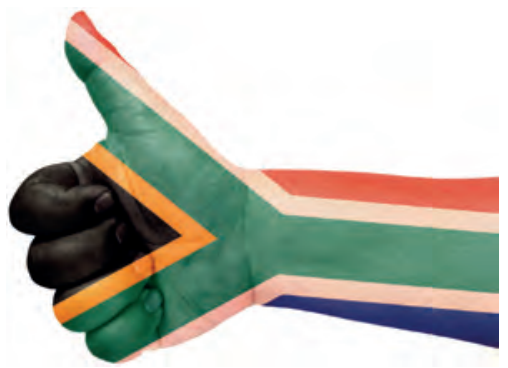

\section{Infos über Südafrika}

\section{$>$ Der Weg zur Freiheit}

Die weißen Südafrikaner stellen nur ca. 8\% der Bevölkerung. Trotzdem gaben sie in den letzten 350 Jahren den Ton an. Um sich ihre Vorrechte und ihren Wohlstand gegenüber der nichtweißen Mehrheit abzusichern, praktizierten sie über Jahrzehnte eine Politik der „Apartheid“ (afrikaans: „Getrenntheit“). Es gab getrennte Schulen für Weiße, Schwarze und „Coloureds“. Gemischte Ehen waren verboten. Sogar die Eingänge zu den öffentlichen Toiletten waren getrennt. Dieses seltsame System führte zu blutigen Ausschreitungen und internationaler Ächtung Südafrikas. Erst Ende der 80er Jahre entsorgten die Südafrikaner - eher widerwillig moderiert vom damaligen Präsidenten Willem de Klerk - die Apartheid auf die Müllhalde der Geschichte. Der Freiheitskämpfer Nelson Mandela wurde 1990 aus dem Gefängnis entlassen und gewann1994 mit dem „African National Congress" die ersten freien Wahlen. Seither stellt der ANC die Regierung, er konnte aber viele Erwartungen nicht erfüllen. Die Korruption grassiert. Armut, Gewalt und schlechte Sicherheitslage prägen den Alltag vieler Menschen.

\section{$>$ Kapstadt}

Im oft euphemistisch als „Regenbogennation" titulierten Südafrika leben viele Ethnien eng beieinander. Besonders bunt ist das Bild in Kapstadt. Neben den „typischen“ Capetonians (Nachkommen von südostasiatischen und afrikanischen Sklaven) leben hier auch viele Mulatten, Schwarze und weiße Südafrikaner. Es gibt eine große muslimische Gemeinde, hinzu kommen Einwanderer aus Europa, den USA, Indien und dem übrigen Afrika. Sehenswürdigkeiten sind der Tafelberg - und die Gefängnisinsel Robben Island ca. 10 km vor Kapstadt im Atlantik. Dort verbrachte Nelson Mandela 18 Jahre hinter Gittern.

Du möchtest in Südafrika ein PJ-Tertial www. oder eine Famulatur absolvieren? Tipps und Infos findest du in unserem Infopaket Südafrika unter: bit.ly/infopaket-suedafrika 